\title{
Light Higgsinos for electroweak naturalness in mirage-mediated high-scale supersymmetry
}

\author{
Kwang Sik Jeong ${ }^{1, *}$ and Chan Beom Park $\oplus^{2, \dagger}$ \\ ${ }^{1}$ Department of Physics, Pusan National University, Busan 46241, South Korea \\ ${ }^{2}$ Center for Theoretical Physics of the Universe, Institute for Basic Science (IBS), \\ Daejeon 34051, South Korea
}

(Received 18 March 2021; accepted 9 December 2021; published 28 December 2021)

\begin{abstract}
Mirage mediation realized in the Kachru-Kallosh-Linde-Trivedi (KKLT) flux compactification can naturally suppress the up-type Higgs soft mass at low-energy scales. As a result, compared to the conventional scenarios, the degree of electroweak fine-tuning can be reduced further up to by a loop factor if the Higgsinos are much lighter than the heavy Higgs doublet. Interestingly, this feature holds even in high-scale supersymmetry as long as the gauge coupling unification, which is required as a prerequisite for mirage mediation, accommodates such light Higgsinos. Under the experimental constraints on the observed Higgs boson, it turns out that mirage mediation can exhibit low electroweak fine-tuning better than a few percent for stops between about $2 \mathrm{TeV}$ and $6 \mathrm{TeV}$, i.e., at the same level as in the weak-scale supersymmetry, if the Higgsinos are around or below a few hundred GeV.
\end{abstract}

DOI: 10.1103/PhysRevD.104.115028

\section{INTRODUCTION}

Supersymmetry (SUSY) is a unique extension of the Poincaré spacetime symmetry, and it has been extensively explored as one of the most plausible candidates for physics beyond the Standard Model (SM) because it can address various problems of the SM such as dark matter, unification, and cosmological inflation [1,2]. In particular, it has been strongly motivated by the hope to explain the huge hierarchy between the weak scale and the unification or Planck scale. However, the current experimental results indicate that SUSY, if realized in nature, should be above the $\mathrm{TeV}$ scale. In this circumstance, as it becomes more difficult to stabilize the electroweak scale against large radiative corrections from unknown ultraviolet physics, one might need to rely on some other mechanism, such as the cosmological relaxation [3], in order to explain the hierarchy between the SUSY breaking scale and the weak scale.

If SUSY exists above the $\mathrm{TeV}$ scale, an important question worth asking is whether it can still solely solve the gauge hierarchy problem, which has been believed to be one of the important virtues of SUSYIn this paper,

\footnotetext{
ksjeong@pusan.ac.kr

cbpark@ibs.re.kr
}

Published by the American Physical Society under the terms of the Creative Commons Attribution 4.0 International license. Further distribution of this work must maintain attribution to the author(s) and the published article's title, journal citation, and DOI. Funded by SCOAP . we explore the possibility of relaxing the electroweak finetuning in high-scale SUSY from mirage mediation [4] that is realized in the KKLT flux compactification $[5,6]$. It has been noticed that $\mathrm{TeV}$ scale mirage mediation can naturally suppress the low-energy value of the up-type Higgs soft mass through the combined effect of anomaly and moduli mediations $[7,8]$. Consequently, it can reduce the degree of fine-tuning for electroweak symmetry breaking roughly by a loop factor if the Higgsinos are much lighter than the heavy Higgs doublet. We stress that this naturalness feature persists even in mirage-mediated high-scale SUSY as long as the gauge coupling unification, which is a prerequisite for mirage mediation, allows the Higgsinos to be very light compared to other sparticles.

Mirage mediation, in which anomaly mediation is comparable to moduli mediation in strength, effectively corresponds to pure moduli mediation transmitted at the mirage messenger scale, where there is no physical threshold. The electroweak fine-tuning can considerably be reduced when the mirage-messenger scale exists around the SUSY breaking scale. It only requires a proper choice of the discrete numbers associated with the string moduli sector $[7,8]$. We find that such a mirage-mediation scheme should make stops heavier than about $2 \mathrm{TeV}$ in order to give the correct mass to the SM-like Higgs boson, but nonetheless, it can exhibit low electroweak fine-tuning better than a few percent for stops below $6 \mathrm{TeV}$ if the Higgsinos are around or below a few hundred GeV. One of the important consequences of mirage mediation is that it leads to highly compressed spectra of gauginos and sfermions, thus allowing us to precisely fix the Higgsino mass by the 
condition of gauge coupling unification within the minimal-supersymmetric SM (MSSM).

This paper is organized as follows. In Sec. II, we present a review on how the Higgs sector parameters are generated in the mirage mediation scheme and then discuss the way to reduce the degree of electroweak fine-tuning in high-scale SUSY while making the SMlike Higgs boson compatible with the current experimental data. The viable parameter region leading to natural electroweak symmetry breaking is examined in Sec. III. The final section is for conclusions.

\section{HIGGS SECTOR IN MIRAGE MEDIATION}

In the MSSM, the Z-boson mass is determined by the minimization condition of the Higgs scalar potential,

$$
\frac{m_{Z}^{2}}{2}=-|\mu|^{2}+\frac{m_{H_{d}}^{2}-m_{H_{u}}^{2} \tan ^{2} \beta}{\tan ^{2} \beta-1},
$$

where the involved parameters in the expression above are evaluated around the weak scale, and $\tan \beta$ is the ratio of the vacuum expectation values (VEVs) of the Higgs doublets, $H_{u}$ and $H_{d}$. The $Z$-boson mass is quite sensitive to the variation of $m_{H_{u}}^{2}$ for moderate to large values of $\tan \beta$, and in general, the electroweak fine-tuning becomes more severe as the SUSY breaking scale increases. Furthermore, the successful electroweak symmetry breaking also requires a sizable Higgs mixing term,

$$
|B \mu|=\frac{\sin 2 \beta}{2}\left(m_{H_{d}}^{2}+m_{H_{u}}^{2}+2|\mu|^{2}\right)
$$

at the weak scale. The degree of fine-tuning quantifies to what extent the $Z$-boson mass is sensitive to the variations of the Higgs sector parameters. As shall be discussed later, the correct estimation of fine-tuning requires the addition of the loop potential to the renormalization group (RG) improved tree-level potential.

Combined with the nonobservation of sparticles around the $\mathrm{TeV}$ scale in collider experiments so far, the measured Higgs boson mass $m_{h} \simeq 125 \mathrm{GeV}$ indicates that SUSY may show up around or above the multi-TeV scale if realized in nature. It is thus generally expected that the electroweak symmetry breaking would require severe finetuning, at the level of $0.1 \%$, unless some other mechanism to cure the fine-tuning is invoked. Having said that, if the Higgsinos are relatively light as compared to other sparticles, the degree of fine tuning can be reduced by a sizable amount in a mediation scheme such that the up-type Higgs soft mass is naturally suppressed at low-energy scales,

$$
\left|m_{H_{u}}^{2}\right| \lesssim|\mu|^{2} \ll m_{H_{d}}^{2} .
$$

In terms of the electroweak fine-tuning, the stop sector is particularly important since it affects the RG running of the up-type Higgs soft mass via the top-quark Yukawa coupling. The stop radiative contribution to $m_{H_{u}}^{2}$ is given by

$$
\delta m_{H_{u}}^{2} \simeq-\frac{3 y_{t}^{2}}{4 \pi^{2}} m_{\tilde{t}}^{2} \ln \left(\frac{\Lambda_{\mathrm{mess}}}{m_{\tilde{t}}}\right),
$$

where $m_{\tilde{t}}$ is the stop mass, and $\Lambda_{\text {mess }}$ denotes the messenger scale at which SUSY breaking is transmitted to the MSSM sector. It shows that having a suppressed value of $m_{H_{u}}^{2}$ below the order of $m_{\tilde{t}}^{2}$ at low energy is unattainable unless the messenger scale is very low. In this regard, miragemediated SUSY breaking, realized in the KKLT flux compactification, is of particular interest because it effectively corresponds to pure moduli mediation transmitted at the mirage messenger scale, $M_{\text {mir }}$, while not being bothered by physical thresholds at the scale. The mirage messenger scale can be very low, depending on the relative strength of anomaly mediation.

It should be noted that the inclusion of the loop potential effectively amounts to the shift, roughly given by

$$
m_{H_{u}}^{2} \rightarrow m_{H_{u}}^{2}-\frac{3 y_{t}^{2}}{8 \pi^{2}} m_{\tilde{t}}^{2},
$$

in the RG-improved tree-level potential. The above correction is quadratically sensitive to the sparticle mass scale and remains unsuppressed in mirage mediation as is the case in other scenarios. The stop contribution (2.4), which generally makes the Higgs mass more sensitive to UV scales due to the logarithmic factor, is the one we wish to suppress within the KKLT framework. The minimization condition (2.1) requires the Higgsino mass parameter roughly to be

$$
|\mu|^{2} \approx \frac{m_{H_{d}}^{2}}{\tan ^{2} \beta}-m_{H_{u}}^{2},
$$

for moderate or large $\tan \beta$. Moreover, the gauge coupling unification is required for the sparticles to exhibit the mirage pattern, and it can be achieved in high-scale SUSY as precisely as in weak-scale SUSY if the Higgsinos are light, as discussed below.

In the KKLT flux compactification, sparticle masses exhibit the mirage mediation pattern as a result of mixed anomaly and moduli mediation. Provided that the gauge couplings unify at $M_{\mathrm{GUT}} \sim 10^{16} \mathrm{GeV}$, the gaugino masses are given by

$$
M_{a}(Q)=M_{0}\left\{1-\frac{b_{a} g_{a}^{2}}{4 \pi^{2}} \ln \left(\frac{M_{\mathrm{mir}}}{Q}\right)\right\},
$$

at the renormalization scale $Q$, with $b_{a}$ being the coefficients of one-loop beta functions. Here the relative strength between anomaly and moduli mediation is measured by the parameter defined as 


$$
\alpha \equiv \frac{m_{3 / 2}}{M_{0} \ln \left(M_{\mathrm{Pl}} / m_{3 / 2}\right)},
$$

with $m_{3 / 2}$ being the gravitino mass. Then, the mirage messenger scale is determined by

$$
M_{\mathrm{mir}}=M_{\mathrm{GUT}}\left(\frac{m_{3 / 2}}{M_{\mathrm{Pl}}}\right)^{\alpha / 2} \text {. }
$$

In the KKLT setup, the $\alpha$ parameter has a positive rational number of order unity at leading order. The soft SUSY breaking parameters of scalar fields also take the mirage pattern

$$
\begin{aligned}
A_{i j k}(Q) & =M_{0}\left\{1+\frac{\gamma_{i}+\gamma_{j}+\gamma_{k}}{8 \pi^{2}} \ln \left(\frac{M_{\mathrm{mir}}}{Q}\right)\right\}, \\
m_{i}^{2}(Q) & =M_{0}^{2}\left\{c_{i}+\frac{1}{4 \pi^{2}}\left(\gamma_{i}-\frac{\dot{\gamma}_{i}}{2} \ln \left(\frac{M_{\mathrm{mir}}}{Q}\right)\right) \ln \left(\frac{M_{\mathrm{mir}}}{Q}\right)\right\},
\end{aligned}
$$

for a proper choice of matter modular weights, where $\gamma_{i}$ is the anomalous dimension of the corresponding field, and the dot denotes differentiation with respect to $Q$. Here $c_{i}$ parametrizes the moduli-mediated contribution, given by

$$
c_{i}=1-n_{i}+\mathcal{O}\left(\frac{1}{8 \pi^{2}}\right) \text {, }
$$

including quantum corrections from string loops and higher-order $\alpha^{\prime}$ corrections. The modular weight $n_{i}$ has a rational number of order unity, depending on the location of the matter in extra dimensions. The above mirage pattern of soft scalar parameters requires the matter fields having a Yukawa coupling $y_{i j k}$ to have modular weights satisfying

$$
n_{i}+n_{j}+n_{k}=2 \text {, }
$$

unless $y_{i j k}^{2}$ is smaller than $1 / 8 \pi^{2}$.

In the generalized KKLT scenario with dilaton-moduli mixing in gauge kinetic functions, the $\alpha$ parameter can have various values, depending on discrete numbers associated with dilaton and moduli couplings [7,8]. Out of this framework, we can have $\alpha=2$ while allowing an extradimensional interpretation for the origin of SUSY breaking. The scenario of mirage mediation with $\alpha=2$ and $n_{H_{u}}=1$ is of particular interest because it can considerably reduce the electroweak fine-tuning. For $\alpha=2$, the mirage messenger scale is fixed at

$$
M_{\mathrm{mir}}=\frac{M_{\mathrm{GUT}}}{M_{\mathrm{Pl}}} m_{3 / 2} \sim M_{0},
$$

and thereby is around the SUSY breaking scale. Here we have used the relation that the ratio between the Planck and unification scales is numerically close to a loop factor. Besides, if $n_{H_{u}}=1$, the up-type Higgs soft mass at $M_{\text {mir }}$ is loop-suppressed relative to the squared soft scalar mass of other scalar fields. Interestingly, the electroweak finetuning remains tempered even for $M_{0}$ being the multi-TeV energy regime as long as the gauge coupling unification is maintained. As a benchmark for accomplishing a natural electroweak symmetry breaking, let us assign matter modular weights as

$n_{H_{u}}=1, \quad n_{H_{d}}=0, \quad n_{\text {quarks }}=n_{\text {leptons }}=\frac{1}{2}$,

where $n_{H_{u}}=1$ is required to reduce the electroweak finetuning, while there is no strict requirement on the modular weights of other fields as long as the mirage conditions (2.12) are satisfied. ${ }^{1}$ For the above choice of modular weights, the mirage conditions are satisfied with good accuracy in the region with $\tan ^{2} \beta \ll\left(m_{t} / m_{b}\right)^{2}$ because the effects of Yukawa couplings (other than that of top quark) can be neglected in the RG evolution of scalar soft parameters. Here we have assumed that the sfermions are supposed to feel SUSY breaking through flavorconserving interactions unless they are heavier than about $100 \mathrm{TeV}$. Note that mirage mediation preserves CP symmetry as a result of axionic shift symmetries associated with the moduli. One can then find that the gaugino masses are universal

$$
M_{a}=M_{0}
$$

and the soft SUSY breaking parameters for scalar fields read

$$
\begin{aligned}
A_{t} & \simeq M_{0}, \\
A_{b} & \simeq A_{\tau} \simeq 2 M_{0}, \\
m_{H_{u}}^{2} & =\mathcal{O}\left(\frac{M_{0}^{2}}{8 \pi^{2}}\right), \\
m_{H_{d}}^{2} & \simeq M_{0}^{2}, \\
m_{\tilde{q}}^{2} & \simeq m_{\tilde{\ell}}^{2} \simeq \frac{M_{0}^{2}}{2},
\end{aligned}
$$

\footnotetext{
${ }^{1}$ A matter field has $n_{i}=0,1 / 2$, or 1 depending on its location in extra dimensions. The modular weight can have a different rational number in a generalized KKLT with an anomalous U(1) gauge symmetry [9]. One may assign different modular weights to the down-type Higgs and matter fields while keeping the flavor universality and satisfying the mirage condition. It can change the degree of electroweak fine-tuning only slightly if the modular weights are of order unity. Still, we need $n_{i}<1$ for quarks and leptons since otherwise there would appear dangerous color or charge-breaking minima. See Ref. [10] for a recent study of other viable assignment for matter modular weights.
} 
at the energy scale $Q=M_{\text {mir }} \sim M_{0}$. Here $\tilde{q}$ and $\tilde{\ell}$ refer to squarks and sleptons, respectively, dropping the flavor indices.

Let us now examine how light the Higgsino can be in the mirage mediation scheme. In the MSSM, the gauge coupling unification can successfully be attained in the absence of heavy threshold corrections if the sparticle spectrum satisfies the following relation $[11,12]^{2}$ :

$\frac{|\mu|}{m_{*}}\left(\frac{m_{H}}{m_{*}}\right)^{1 / 4}\left(\frac{M_{2}}{m_{*}}\right)^{1 / 3}\left(\frac{M_{2}}{M_{3}}\right)^{7 / 3}\left(\frac{m_{\tilde{\ell}}}{m_{\tilde{q}}}\right)^{1 / 4}=1$,

with $m_{H}$ being the heavy Higgs doublet mass. The scale parameter $m_{*}$ determines the precision of the gauge coupling unification. It should be above a few hundred $\mathrm{GeV}$ and below $10 \mathrm{TeV}$ to achieve the unification within a deviation of a few percent. As a consequence of the relation, for mirage mediation with $\alpha=2$ and the modular weights given by the relation (2.14), we find the Higgsino mass as

$$
|\mu| \simeq 130 \mathrm{GeV}\left(\frac{m_{*}}{0.5 \mathrm{TeV}}\right)^{19 / 12}\left(\frac{M_{0}}{5 \mathrm{TeV}}\right)^{-7 / 12},
$$

assuring the gauge coupling unification at $M_{\mathrm{GUT}} \sim 10^{16} \mathrm{GeV}$. Therefore, mirage mediation can be realized in high-scale SUSY while accommodating light Higgsinos, even around the weak scale. In high-scale SUSY with light Higgsinos, the lightest neutralinos and the lightest chargino are dominated by the Higgsino components, and their mass difference is estimated by

$$
\begin{aligned}
\Delta m= & m_{\chi_{1}^{+}}-m_{\chi_{1}^{0}} \simeq 0.8 \mathrm{GeV}\left(\frac{M_{0}}{5 \mathrm{TeV}}\right)^{-1} \\
& +0.3 \mathrm{GeV}\left(\frac{|\mu|}{300 \mathrm{GeV}}\right)^{0.15},
\end{aligned}
$$

including the contributions from gauge boson loops [14]. Here we have used the sparticle mass pattern of mirage mediation with $\alpha=2$, where the gauginos have a degenerate mass spectrum, $M_{a} \simeq M_{0}$. Although it would be difficult to detect the lightest chargino at the LHC because of the degenerate mass spectrum, we expect that future lepton colliders may probe the signals from the processes of $e^{+} e^{-} \rightarrow \chi_{1}^{0} \chi_{2}^{0} \gamma$ or $\chi_{1}^{+} \chi_{1}^{-} \gamma$, mediated by a virtual $Z$ boson or photon $[15,16]$.

We close this section by discussing the dynamical generation of the Higgs mixing parameter. In models with

\footnotetext{
${ }^{2}$ If high-scale threshold corrections are sizable, the gauge coupling unification can accommodate a much wider spectrum of sparticle masses [13], allowing for lighter Higgsinos than required by the relation (2.17). In such a case, however, the mirage-mediated pattern of sparticle masses is spoiled, making it difficult to suppress the up-type Higgs soft mass at low-energy scales. See Appendix for details.
}

sizable anomaly mediation, the Higgs mixing parameter is generally of the order of the gravitino mass, which is too large to induce electroweak symmetry breaking correctly. To generate it at the right scale, i.e., to have $B \sim M_{0}$, one can extend the Higgs sector, for instance, by coupling it to the Kähler modulus $T$ through the nonperturbative superpotential term from hidden gaugino condensation

$$
\Delta W=A e^{-\frac{1}{2} a T} H_{u} H_{d},
$$

for $T$ stabilized by the nonperturbative superpotential term, $\Delta W_{\mathrm{np}}=A_{0} e^{-a T}$, in the KKLT setup [7,8]. Another interesting way to obtain $B \sim M_{0}$ is to consider the effective Kähler potential

$$
\Delta K=\kappa \frac{\bar{S}}{S} H_{u} H_{d}+\text { H.c. },
$$

in the model, where the singlet scalar $S$ is radiatively stabilized $[17,18]$. In this case, the phase component of $S$ can play the role of the axion that can provide a solution to the strong $C P$ problem. It is also worthwhile to note that the fermionic partner, the axino, can contribute to the dark matter of the Universe while avoiding the cosmological problems arising when the Universe experiences the modulus-dominated phase [17].

\section{ELECTROWEAK NATURALNESS}

In this section we examine how naturally the electroweak symmetry breaking arises in the mirage mediation with $\alpha=2$ and $n_{H_{u}}=1$. In this benchmark scenario, the Higgs soft masses are given by

$$
\left|m_{H_{u}}^{2}\right| \sim \frac{m_{\tilde{t}}^{2}}{8 \pi^{2}} \ll m_{H_{d}}^{2} \sim m_{\tilde{t}}^{2},
$$

at the SUSY breaking scale, with the stop mass $m_{\tilde{t}} \simeq$ $M_{0} / \sqrt{2}$ as presented in the relation (2.16). The value of $m_{H_{u}}^{2}$ is rather sensitive to the renormalization scale at energy scales around the mirage messenger scale, which is close to the stop mass $m_{\tilde{t}}$. To cancel the dependence of the Higgs sector parameters on the renormalization scale, one needs to include one-loop effective potential as

$$
V=V_{\text {tree }}+\Delta V
$$

where $V_{\text {tree }}$ is the RG improved tree-level potential, and the loop potential $\Delta V$ is generated dominantly by the loops involving third-generation sfermions. In the electroweak symmetry breaking conditions derived from $V_{\text {tree }}$, the inclusion of $\Delta V$ effectively corresponds to the replacement

$$
m_{i}^{2} \rightarrow m_{i}^{2}+t_{i}
$$


for $i \in\left\{H_{u}, H_{d}\right\}$. Here the tadpoles $t_{i}$ are calculable from the squared mass matrix after electroweak symmetry breaking [19], and have the values of

$$
\left|t_{i}\right| \sim \frac{M_{0}^{2}}{8 \pi^{2}}
$$

The above radiative corrections represent the quadratic sensitivity of the Higgs mass to the scale of sparticle masses. Our scenario is to suppress the RG evolved up-type Higgs mass squared down to the order of the tadpoles, which is naturally achieved by taking $\alpha=2$ and $n_{H_{u}}=1$ in mirage mediation. As a result, the degree of electroweak fine-tuning can be reduced further, up to a loop factor, compared to the conventional scenarios.

For the correct estimation of the degree of electroweak fine-tuning, one should note that the Higgs mass parameters are given by $m_{i}^{2}=c_{i} M_{0}^{2}$ at the mirage messenger scale, in which $c_{i}$ generally does receive model-dependent quantum corrections from string loops and higher-order $\alpha^{\prime}$ corrections. This implies that the RG improved Higgs mass parameters include a correction of the order of $M_{0}^{2} / 8 \pi^{2}$, thus it is as sizable as the tadpoles $t_{i}$ from the loop potential. Taking this into account, we examine the electroweak symmetry breaking using $V_{\text {tree }}$ by replacing the Higgs mass parameters as

$$
m_{i}^{2} \rightarrow \tilde{m}_{i}^{2}=\left(1-n_{i}+\frac{\kappa_{i}}{8 \pi^{2}}\right) M_{0}^{2}
$$

for constants $\kappa_{i}$ of order unity or below. The parameters $\kappa_{i}$ encompass all the renormalization scale dependence and higher-order effects; the tadpole $t_{i}$, higher-order moduli mediated contributions, and model-dependent stringy higher-order corrections. It is obvious that $\kappa_{i}$ should be treated as a free parameter of order unity or below due to the stringy corrections. We emphasize that setting $\kappa_{H_{u}}$ to be a free parameter is quite important in our scenario because $n_{H_{u}}=1$ gives $\tilde{m}_{H_{u}}^{2}=\kappa_{H_{u}} M_{0}^{2} / 8 \pi^{2}$. In our analysis below, the value of $\kappa_{H_{u}}$ is fixed by imposing the minimization condition of the Higgs potential.

Let us now explore the parameter region leading to the correct electroweak symmetry breaking while satisfying the current experimental constraints. The minimization conditions now read

$$
\begin{aligned}
\frac{1}{2} m_{Z}^{2} & =-|\mu|^{2}+\frac{\tilde{m}_{H_{d}}^{2}-\tilde{m}_{H_{u}}^{2} \tan ^{2} \beta}{\tan ^{2} \beta-1}, \\
\sin 2 \beta & =\frac{2|B \mu|}{\tilde{m}_{H_{d}}^{2}+\tilde{m}_{H_{u}}^{2}+2|\mu|^{2}},
\end{aligned}
$$

with the Higgs mass parameters given by

$$
\begin{aligned}
& \tilde{m}_{H_{u}}^{2}=\frac{\kappa_{H_{u}}}{8 \pi^{2}} M_{0}^{2}, \\
& \tilde{m}_{H_{d}}^{2}=\left(1+\frac{\kappa_{H_{d}}}{8 \pi^{2}}\right) M_{0}^{2} .
\end{aligned}
$$

Here the Higgs sector parameters must satisfy

$$
\begin{aligned}
\tilde{m}_{H_{d}}^{2}+\tilde{m}_{H_{u}}^{2}+2|\mu|^{2} & >2|B \mu|, \\
\left(\tilde{m}_{H_{d}}^{2}+|\mu|^{2}\right)\left(\tilde{m}_{H_{u}}^{2}+|\mu|^{2}\right) & <|B \mu|^{2},
\end{aligned}
$$

for the scalar potential to be bounded from below and to have a minimum at nonzero Higgs VEVs. The minimization conditions are insensitive to the value of $\kappa_{H_{d}}$, so we will simply set $\kappa_{H_{d}}=0$ in the numerical analysis. The viable parameter region can then be examined by scanning over the two-dimensional space of

$$
\left\{M_{0}, \tan \beta\right\} .
$$

The value of $\mu$ is fixed by the unification condition (2.18) for a given value of $m_{*}$, and the $B$ and $\kappa_{H_{u}}$ values are obtained by the minimization conditions (3.6).

At present, one of the most important constraints on models with an extended Higgs sector is from the measurement of the Higgs boson mass. The viable parameter region of $\left\{M_{0}, \tan \beta\right\}$ can thus be found by requiring the SM-like Higgs boson to have $m_{h} \simeq 125 \mathrm{GeV}$. In the MSSM, the mass of the lightest neutral Higgs boson reads

$$
\begin{aligned}
\left.m_{h}^{2}\right|_{\text {tree }} & =\frac{1}{2}\left(m_{Z}^{2}+m_{A}^{2}-\sqrt{\left(m_{Z}^{2}+m_{A}^{2}\right)^{2}-4 m_{Z}^{2} m_{A}^{2} \cos ^{2} 2 \beta}\right) \\
& \simeq m_{Z}^{2} \cos ^{2} 2 \beta
\end{aligned}
$$

at tree level. The last approximation holds in the decoupling limit with $m_{A} \gg m_{Z}$. Higher-order corrections to the Higgs boson mass arise mainly via the loops of third-generation sfermions, and the gluinos also take part in at two-loop level. Using the effective field theory approach with RG improvements, one finds the Higgs boson mass in the MSSM to be

$$
m_{h}^{2}=\left.m_{h}^{2}\right|_{\text {tree }}+\frac{3 r}{4 \pi^{2}} \frac{\bar{m}_{t}^{4}}{v^{2}},
$$

where $r$ is given by

$$
\begin{aligned}
r= & t+\frac{X_{t}^{2}}{M_{S}^{2}}\left(1-\frac{X_{t}^{2}}{12 M_{S}^{2}}\right)+\left(\frac{4 \alpha_{s}}{3 \pi}-\frac{5 \bar{m}_{t}^{2}}{16 \pi^{2} v^{2}}\right) t \\
& +\frac{1}{16 \pi^{2}}\left(\frac{3}{2} \frac{\bar{m}_{t}^{2}}{v^{2}}-32 \pi \alpha_{s}\right)\left\{\frac{X_{t}^{2}}{M_{S}^{2}}\left(2-\frac{X_{t}^{2}}{6 M_{S}^{2}} t+t^{2}\right)\right\},
\end{aligned}
$$


up to two-loop leading corrections [20-22], with $v \simeq$ $174 \mathrm{GeV}$ and $t \equiv \ln \left(M_{S}^{2} / \bar{m}_{t}^{2}\right)$. Here $M_{S}^{2}$ is the average of the squared masses of two stops, and $X_{t}$ is the stop mixing parameter defined by

$$
X_{t}=A_{t}-\frac{\mu}{\tan \beta}
$$

with $A_{t}$ being the stop trilinear coupling. In the mirage mediation under consideration, the stop sector has

$$
A_{t} \simeq M_{0}, \quad M_{S} \simeq \frac{M_{0}}{\sqrt{2}},
$$

because the modular weights have been assigned to satisfy the mirage conditions. In our analysis, we have evaluated all the couplings at the running top mass $\bar{m}_{t} \simeq 163 \mathrm{GeV}$ in the $\overline{\mathrm{MS}}$ scheme. We have also included the loop contributions from the sbottom and stau adopting the results from Refs. [23,24]. The analytic expression (3.11) is valid if $\tan \beta$ is moderate or large, and if the splitting of the stop mass eigenvalues is small [25]

$$
\frac{m_{\tilde{t}_{2}}^{2}-m_{\tilde{t}_{1}}^{2}}{m_{\tilde{t}_{2}}^{2}+m_{\tilde{t}_{1}}^{2}} \lesssim 0.5 .
$$

We have confirmed that the parameter space considered in our analysis satisfies the above condition.

In Fig. 1, we display the Higgs boson mass as a function of the SUSY breaking scale, $m_{h}\left(M_{0}\right)$, for the mirage mediation with soft SUSY-breaking terms given by

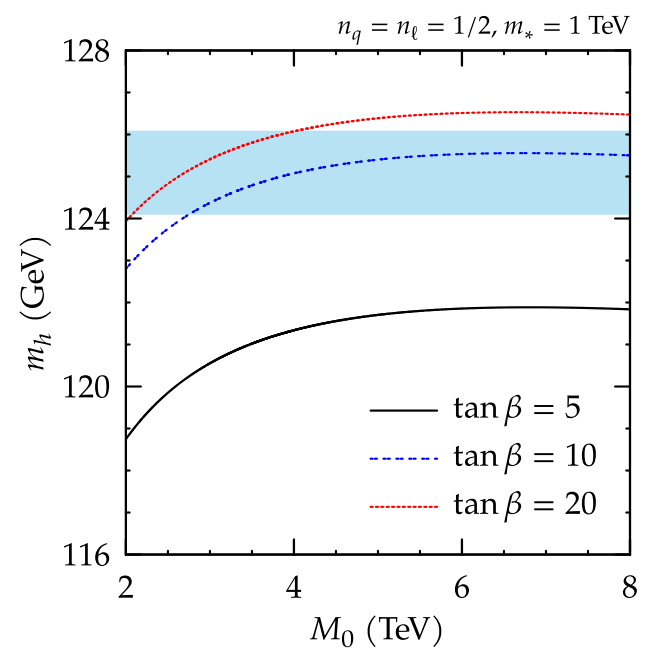

FIG. 1. Mass of the SM-like Higgs boson as a function of the SUSY breaking scale $M_{0}$. Here we have fixed the Higgsino mass parameter $\mu$ under the condition of precise gauge coupling unification by taking the SUSY threshold scale to be $m_{*}=1 \mathrm{TeV}$. The blue-shaded band corresponds to $m_{h}=125.09 \pm 1 \mathrm{GeV}$.
(2.15) and (2.16) at the mirage messenger scale $M_{\text {mir }} \sim M_{0}$. Note that the Higgs sector parameters, $\mu, B$, and $m_{H_{u}}^{2}$ are fixed by the unification condition (2.18) and the minimization conditions, respectively. For large $M_{0}$, the stop mixing is sizable but smaller than the maximal mixing where $\left|X_{t}\right|=\sqrt{6} M_{S}$. Taking into account the fact that light Higgsinos are favored for reducing the electroweak fine-tuning, we have taken the SUSY threshold scale to be $m_{*}=1 \mathrm{TeV}$ to fix the value of $\mu$ by the unification condition. We will discuss the effect of the SUSY threshold scale shortly. Currently, the uncertainty of the combined measurement of the Higgs boson mass from the ATLAS and CMS experiments at the LHC is $0.24 \mathrm{GeV}$ [26]. However, because the theoretical uncertainty of the Higgs mass calculation in the SUSY models is typically about a few $\mathrm{GeV}$ [27-29], we show the Higgs boson mass lying in the range of $m_{h}=125.09 \pm 1 \mathrm{GeV}$ in the figure. From the numerical analysis, we find that $\tan \beta$ should be large enough to have $m_{h}$ around the measured value. For $m_{*}=1 \mathrm{TeV}$, the lower bound is numerically found to be $\tan \beta \gtrsim 8$. One can also see that a larger $M_{0}$ value is required for smaller $\tan \beta$.

The values of $M_{0}$ and $\tan \beta$ leading to the correct Higgs boson mass can be seen in Fig. 2. Again, the blue-shaded region corresponds to the parameter space of $m_{h}=125.09 \pm 1 \mathrm{GeV}$ in each panel. It shows that the $M_{0}$ value tends to be larger for smaller $\tan \beta$. For $m_{h}=$ $125 \mathrm{GeV}$ and $\tan \beta=10$, we need $M_{0} \simeq 4 \mathrm{TeV}$. In the figure, we have taken $m_{*}=0.5 \mathrm{TeV}$ and $1 \mathrm{TeV}$ in the left and right panels, respectively. The $m_{*}$ value does not affect the $M_{0}$ value but it does affect model parameters, such as $B$, by changing the Higgsino mass parameter through the condition of gauge coupling unification as given in Eq. (2.18). In most of the parameter space, we find that $|B|$ is around or below $M_{0}$, which lies in the range expected in the models as discussed in the previous section. For $m_{*}=1 \mathrm{TeV}$ and $\tan \beta=10,|B| \simeq M_{0}$. The experimental constraint on the $M_{0}$ value comes from the gluino searches at the LHCCurrently, the lower bound on the gluino mass is about $2.2 \mathrm{TeV}$ [30,31]. In Fig. 2, one can see that the region with large $\tan \beta$ already starts to be excluded due to the gluino bound. The $M_{0}$ parameter also receives constraints from stop searches at the LHC [32,33], but the corresponding bound is weaker than that from the gluino searches; $M_{0} \gtrsim 1.6 \mathrm{TeV}$ for $m_{\tilde{t}_{1}}>1 \mathrm{TeV}$.

As the benchmark points of the $\mathrm{TeV}$ scale mirage mediation with $\alpha=2$ and $n_{H_{u}}=1$, we present the Higgs and sparticle mass spectra for the input parameters at $Q=$ $M_{\text {mir }}$ in Table I. The mass spectra have been calculated with SOFTSUSY [34]. The soft SUSY-breaking parameters are set by the mirage relations given in (2.15) and (2.16), and the Higgsino mass parameter $\mu$ is fixed by the condition of the gauge coupling unification in (2.17) with $m_{*}=1 \mathrm{TeV}$. We have taken $\mu>0$ and $m_{t}=173 \mathrm{GeV}$. The mirage 

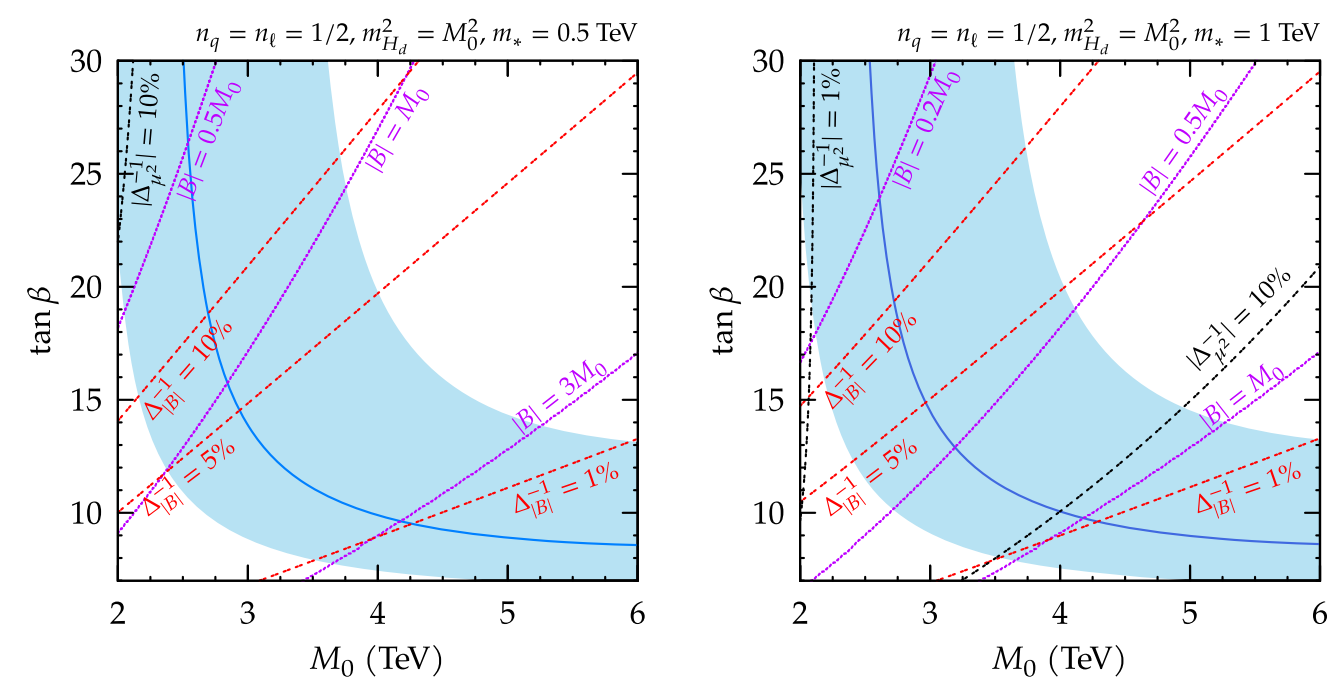

FIG. 2. Parameter space of $M_{0}$ and $\tan \beta$ consistent with the Higgs boson mass. for $m_{*}=0.5 \mathrm{TeV}$ (left) and $1 \mathrm{TeV}$ (right). The contours of the degrees of fine-tuning for the EWSB are also exhibited. See the text for the details. The blue-shaded region corresponds to $m_{h}=125.09 \pm 1 \mathrm{GeV}$.

messenger scale $M_{\text {mir }}$ is determined as in (2.9) for the gravitino mass $m_{3 / 2}$ obtained using the relation (2.8) and $M_{\mathrm{GUT}}=10^{16} \mathrm{GeV}$. For illustration, $M_{\text {mir }}$ and $m_{3 / 2}$ are also shown in Table I. We note that in the benchmark point with smaller $M_{0}$, the gluino mass is right within the reach of the high-luminosity LHC for an integrate luminosity of $3 \mathrm{ab}^{-1}$ [35], while in the other benchmark point with larger $M_{0}$, it is far beyond the reach.
Let us continue to discuss the fine-tuning issue. In order to estimate quantitatively to what extent the $Z$-boson mass is sensitive to the variations of the Higgs sector parameters, we take the conventional fine-tuning measure defined by

$$
\Delta_{a} \equiv \frac{\partial \ln m_{Z}^{2}}{\partial \ln a}
$$

TABLE I. Higgs and sparticle mass spectra in GeV for (a) smaller and (b) larger $M_{0}$. The first and second generation sfermions are degenerate in mass; $m_{c_{i}} \simeq m_{u_{i}}, m_{s_{i}} \simeq m_{d_{i}}$, and $m_{\mu_{i}} \simeq m_{e_{i}}$ for $i=L, R$. The neutralino masses are taken to be positive. In the bottom rows, we exhibit the values of the fine-tuning measures for each point.

\begin{tabular}{lcc}
\hline \hline Parameters & $(\mathrm{a})$ & $(\mathrm{b})$ \\
\hline$M_{0}(\mathrm{GeV})$ & 2800 & 4000 \\
$\mu(\mathrm{GeV})$ & 548 & 445 \\
$\tan \beta$ & 25 & 10 \\
$M_{\text {mir }}(\mathrm{GeV})$ & 700 & 1000 \\
$m_{3 / 2}(\mathrm{TeV})$ & 169.65 & 272.34 \\
$m_{h}$ & 125.1 & 125.6 \\
$m_{H}, m_{A}, m_{H^{+}}$ & $2855,2855,2856$ & $4044,4044,4045$ \\
$m_{\tilde{g}}$ & 2686 & 3842 \\
$m_{\tilde{\chi}_{1}^{0}}, m_{\tilde{\chi}_{2}^{0}}, m_{\tilde{\chi}_{3}^{0}}, m_{\tilde{\chi}_{4}^{0}}$ & $558,560,2788,2818$ & $455,457,3981,4024$ \\
$m_{\tilde{\chi}_{1}^{+}}, m_{\tilde{\chi}_{2}^{+}}$ & 559,2788 & 456,3981 \\
$m_{\tilde{t}_{1}}, m_{\tilde{t}_{2}}$ & 1796,2030 & 2609,2839 \\
$m_{\tilde{b}_{1}}, m_{\tilde{b}_{2}}$ & 1912,1940 & 2714,2735 \\
$m_{\tilde{u}_{L}}, m_{\tilde{u}_{R}}, m_{\tilde{d}_{L}}, m_{\tilde{d}_{R}}$ & $1890,1905,1891,1905$ & $2704,2726,2705,2724$ \\
$m_{\tilde{\tau}_{1}}, m_{\tilde{\tau}_{2}}$ & 1973,1991 & 2808,2819 \\
$m_{\tilde{e}_{L}}, m_{\tilde{e}_{R}}$ & 1964,1970 & 2806,2815 \\
$\Delta_{|\mu|^{2}}, \Delta_{|B|}, \Delta_{m_{H_{d}}^{2}}, \Delta_{m_{H_{u}}^{2}}$ & $-69.7,6.3,-3.0,70.5$ & $-9.5,80.3,-39.7,10.1$ \\
$\Delta_{M_{0}^{2}}, \Delta_{\kappa_{H_{d}}}, \Delta_{\kappa_{H_{u}}}$ & $67.5,0,70.6$ & $-29.6,0,10.1$ \\
\hline \hline
\end{tabular}


where $a$ stands for the parameters involved in the Higgs scalar potential [36-38]. ${ }^{3}$ For moderate to large $\tan \beta$ values, the ratio between the Higgs VEVs is given by $\tan \beta \simeq\left(\tilde{m}_{H_{d}}^{2}+\tilde{m}_{H_{u}}^{2}+2|\mu|^{2}\right) /|B \mu|$. Then, in terms of the mass parameters in the Higgs sector, $a=\left\{\mu, B, \tilde{m}_{H_{d}}^{2}\right.$, $\left.\tilde{m}_{H_{u}}^{2}\right\}$, the $Z$-boson mass is expressed as follows:

$$
\frac{1}{2} m_{Z}^{2} \simeq \frac{|B \mu|^{2} \tilde{m}_{H_{d}}^{2}}{\left(\tilde{m}_{H_{d}}^{2}+\tilde{m}_{H_{u}}^{2}+2|\mu|^{2}\right)^{2}}-\tilde{m}_{H_{u}}^{2}-|\mu|^{2} .
$$

In mirage mediation, the soft Higgs masses have the relation that $\left|\tilde{m}_{H_{u}}^{2}\right| \lesssim \tilde{m}_{H_{d}}^{2} / 8 \pi^{2}$, which implies that the minimization conditions of the Higgs scalar potential can be satisfied only for

$$
|\mu|^{2}, \quad\left|\tilde{m}_{H_{u}}^{2}\right| \ll \tilde{m}_{H_{d}}^{2} .
$$

From Eq. (3.17), it is thus straightforward to see that the fine-tuning measures for the $\mu$ and $B$ parameters are given as

$$
\begin{aligned}
& \Delta_{\mu^{2}} \simeq \frac{2|B \mu|^{2}}{m_{Z}^{2} \tilde{m}_{H_{d}}^{2}}-\frac{2|\mu|^{2}}{m_{Z}^{2}} \simeq \frac{2 \tilde{m}_{H_{u}}^{2}}{m_{Z}^{2}}, \\
& \Delta_{|B|} \simeq \frac{4|B \mu|^{2}}{m_{Z}^{2} \tilde{m}_{H_{d}}^{2}},
\end{aligned}
$$

while those for $m_{H_{u}}^{2}$ and $m_{H_{d}}^{2}$ are

$$
\begin{aligned}
& \Delta_{m_{H_{d}}^{2}} \simeq-\frac{2|B \mu|^{2}}{m_{Z}^{2} \tilde{m}_{H_{d}}^{2}} \simeq-\frac{1}{2} \Delta_{|B|}, \\
& \Delta_{m_{H_{u}}^{2}} \simeq-\frac{2 \tilde{m}_{H_{u}}^{2}}{m_{Z}^{2}} \simeq-\Delta_{\mu^{2}},
\end{aligned}
$$

where the last equality in $\Delta_{\mu^{2}}$ follows from the observation that the minimization conditions approximately require $|B \mu|^{2} \simeq\left(\tilde{m}_{H_{u}}^{2}+|\mu|^{2}\right) \tilde{m}_{H_{d}}^{2}$. Therefore, the degree of fine tuning is determined by the larger of $\left|\Delta_{\mu^{2}}\right|$ and $\Delta_{|B|}$. The $Z$-boson mass is more sensitive to the variation of the Higgs mixing parameter $B$,

$$
\Delta_{|B|}^{-1} \simeq 0.05 \times\left(\frac{\tan \beta}{15}\right)^{2}\left(\frac{M_{0}}{3 \mathrm{TeV}}\right)^{-2},
$$

if $|\mu|$ is smaller than $3|B| / \tan \beta$, i.e., if the Higgsinos are as light as

\footnotetext{
${ }^{3}$ There are also other ways to quantify the degree of fine-tuning for the electroweak symmetry breaking. See, for example, Ref. [39] for more discussion. We also refer the reader to Refs. [40,41] for a recent discussion of electroweak naturalness using other convention of the fine-tuning measure in general mirage mediation.
}

$$
|\mu|<346 \mathrm{GeV} \times\left(\frac{\tan \beta}{15}\right)^{-1}\left(\frac{M_{0}}{3 \mathrm{TeV}}\right)
$$

where we have used the minimization condition, $|B \mu| \simeq \tilde{m}_{H_{d}}^{2} / \tan \beta$, with $\tilde{m}_{H_{d}}^{2} \simeq M_{0}^{2}$. Figure 2 shows the contours of the fine-tuning measures as well. We find that the degree of fine-tuning is about a few percent or better in the parameter space of $2 \mathrm{TeV} \lesssim M_{0} \lesssim 6 \mathrm{TeV}$ and $8 \lesssim \tan \beta \lesssim 25$, while being consistent with the measured Higgs boson mass. Multi-TeV SUSY in the mirage mediation can therefore achieve the electroweak symmetry breaking as naturally as the weak-scale SUSY.

The fine-tuning measures (3.19) and (3.20) have been obtained by varying the Higgs sector mass couplings at the weak scale, which are determined by the input parameters, $M_{0}, M_{\text {mir }}, n_{i}$, and $\kappa_{i}$. One may be concerned about the sensitivity of the $Z$-boson mass to the variation of the input parameters and severer fine-tuning than the above estimation. It is, however, not the case, as one can see from

$$
\begin{aligned}
& \Delta_{M_{0}^{2}}=\Delta_{m_{H_{d}}^{2}}+\Delta_{m_{H_{u}}^{2}}, \\
& \Delta_{\kappa_{H_{d}}} \simeq \frac{\kappa_{H_{d}}}{8 \pi^{2}} \Delta_{m_{H_{d}}^{2}}, \\
& \Delta_{\kappa_{H_{u}}}=\Delta_{m_{H_{u}}^{2}},
\end{aligned}
$$

where we have used that $\kappa_{i}$ are of the order unity, and the modular weights are assigned by $n_{H_{u}}=1$ and $n_{H_{d}}=0$. In Table I, we exhibit the values of $\Delta_{M_{0}^{2}}$ and $\Delta_{\kappa_{H_{d}}}$, and $\Delta_{\kappa_{H_{u}}}$ for the benchmark points of mirage mediation with $\alpha=2$ and $n_{H_{u}}=1$. For the variation of $n_{i}$ and $M_{\text {mir }}$, we note that the modular weights $n_{i}$ are not continuous but rational numbers, $0,1 / 2$, or 1 , determined by the location of the corresponding matter in extra dimensions. This implies that the choice of modular weights is not a fine-tuning. The $\alpha$ parameters is also a rational number, and taking $\alpha=2$ leads to

$$
M_{\mathrm{mir}} \simeq \frac{2 M_{\mathrm{GUT}}}{M_{\mathrm{Pl}}} \ln \left(\frac{M_{\mathrm{Pl}} / M_{0}}{2 \ln \left(M_{\mathrm{Pl}} / M_{0}\right)}\right) \times M_{0} .
$$

Here, the unification scale is given by $M_{\mathrm{GUT}} \approx 10^{16} \mathrm{GeV}$, which is insensitive to the value of $M_{0}$ because it is determined by the ratios of the sparticle masses, i.e., by the choice of modular weights. The above expression shows that $M_{\text {mir }}$ is fixed by the $M_{0}$ value. Specifically, it is approximately proportional to $M_{0}$, e.g., $M_{\text {mir }} \approx 0.24 M_{0}$ for $M_{0}$ between $\mathrm{TeV}$ and $\mathrm{PeV}$ scales. The mirage messenger scale appears in soft SUSY breaking couplings, renormalized at the SUSY breaking scale only through the combination of $\ln \left(M_{\text {mir }} / M_{0}\right)$, which rarely changes under the variation of $M_{0}$ for $\alpha=2$. As is well known, the stop sector can significantly affect the $Z$-boson mass through the RG running and loop-potential contributions to the up-type Higgs soft mass squared, (2.4) and (2.5). In mirage 



FIG. 3. Parameter space of $\mu$ and $\tan \beta$ consistent with the Higgs boson mass for $m_{*}=0.5 \mathrm{TeV}$ (left) and $1 \mathrm{TeV}$ (right). The blueshaded region corresponds to $m_{h}=125.09 \pm 1 \mathrm{GeV}$.

mediation, the contributions are proportional to $m_{\tilde{t}}^{2} \ln \left(M_{\text {mir }} / m_{\tilde{t}}\right)$ and $m_{\tilde{t}}^{2}$, respectively, with the stop mass given by $m_{\tilde{t}} \simeq \sqrt{1-n_{q}} M_{0}$. It is therefore obvious that in the case of $\alpha=2$, both the stop contributions are of the order of $M_{0}^{2} / 8 \pi^{2}$, and they are sensitive only to the variation of $M_{0}$. The effects are included in $\Delta_{M_{0}^{2}}$, or equivalently, in $\Delta_{m_{H_{u}}^{2}}$. These observations explain why the degree of finetuning can be reduced up to a loop factor, compared to the conventional scenarios.

We finally discuss the effect of the SUSY threshold scale. A precise gauge coupling unification is achieved for $m_{*} \simeq 2 \mathrm{TeV}$, assuming that the threshold corrections induced by heavy fields of a grand unified theory are absent [11]. If one allows a $3 \%$ deviation of the strong coupling [42], $m_{*}$ could be as small as $200 \mathrm{GeV}$. Setting $m_{*}=1 \mathrm{TeV}$ gives rise to about $1 \%$ deviation. We note that the size of the $\mu$ parameter increases as $m_{*}$ becomes larger, as can be seen in Eq. (2.18). Figure 3 shows the parameter space of $|\mu|$ and $\tan \beta$ consistent with the measured Higgs mass. One important experimental constraint concerning the $\mu$ parameter is the lower bound on the charged Higgsino mass set by the LEP experiment; $|\mu|>104 \mathrm{GeV}$. The bound can be translated into the lower bound on $m_{*}$, which is $m_{*} \gtrsim 300 \mathrm{GeV}$ for $M_{0} \gtrsim 2 \mathrm{TeV}$. Meanwhile, a large $m_{*} \gtrsim 1.5 \mathrm{TeV}$ gives rise to $|\mu| \gtrsim 1 \mathrm{TeV}$, which results in too severe electroweak fine-tuning induced by the $\mu$ parameter; $\left|\Delta_{\mu^{2}}^{-1}\right| \lesssim 0.01$. Therefore, it can be argued that $m_{*}$ is bounded from above by the naturalness of the Higgs sector. We conclude that the SUSY threshold scale of about $300 \mathrm{GeV}$ to a $\mathrm{TeV}$ is consistent with the successful gauge coupling unification within a deviation below a few percent while satisfying the mass bound on the charged Higgsino and the electroweak naturalness.

\section{CONCLUSIONS}

Combined with the null results of SUSY searches at collider experiments so far, the Higgs boson at $125 \mathrm{GeV}$ indicates that SUSY may exist around or above the multi$\mathrm{TeV}$ scale. However, even in such a high-scale SUSY scenario, the electroweak symmetry breaking can naturally occur with low fine-tuning if the sparticles obtain masses via mirage mediation as in the KKLT flux compactification. It is because the up-type Higgs soft mass can be suppressed at low-energy scales, insensitively to the SUSY breaking scale, and light Higgsinos much below the SUSY breaking scale are compatible with the gauge coupling unification. We note that, if the Higgsinos are around or below a few hundred $\mathrm{GeV}$, mirage mediation can serve lowelectroweak fine-tuning better than a few percent for stops between about $2 \mathrm{TeV}$ and $6 \mathrm{TeV}$, while accommodating the Higgs boson consistent with the current experimental data. We expect that the electroweak naturalness with light Higgsinos will be tested by searching for the light neutralinos and charginos at future colliders.

\section{ACKNOWLEDGMENTS}

This work was supported by IBS under the Project Code No. IBS-R018-D1 (C. B. P.), and by the National Research Foundation of Korea (NRF) funded by the Korea government Grants No. 2018R1C1B6006061 and No. 2021R1A4A5031460 (K. S. J.).

\section{APPENDIX: GAUGE THRESHOLD CORRECTIONS}

In this Appendix we examine how much the mirage mediation pattern is affected by gauge threshold corrections. Let us suppose that the gauge couplings receive 
threshold corrections at $m_{\text {th }}$, and consequently do not unify at $M_{\mathrm{GUT}}$ if extrapolated with the MSSM beta functions for running. At energy scales below $m_{\text {th }}$, the gauge couplings are written as

$$
\frac{1}{g_{a}^{2}(Q)}=\frac{1}{g_{0}^{2}}+\frac{b_{a}}{4 \pi^{2}} \ln \left(\frac{M_{\mathrm{GUT}}}{Q}\right)+\Delta_{a},
$$

where $\Delta_{a}$ is the threshold correction to the gauge coupling, and the universal part is determined by the gauge kinetic function, $g_{0}^{2}=1 / \operatorname{Re}\left(f_{a}\right)$, with $f_{a}=T+$ constant. Using the modulus dependence of gauge couplings, one finds the gaugino masses to be

$$
M_{a}\left(m_{\mathrm{th}}\right)=\frac{g_{a}^{2}\left(m_{\mathrm{th}}\right)}{g_{0}^{2}} M_{0}+\frac{b_{a} g_{a}^{2}\left(m_{\mathrm{th}}\right)}{8 \pi^{2}} m_{3 / 2},
$$

at the scale just below $m_{\mathrm{th}}$. Here the term proportional to $b_{a}$ is the anomaly-mediated contribution, which does not depend on physics at higher-energy scales. Because the combination $M_{a} / g_{a}^{2}$ does not run with the scale, low-energy gaugino masses are obtained by

$$
M_{a}(Q)=M_{a 0}\left\{1-\frac{b_{a} g_{a}^{2}(Q)}{4 \pi^{2}} \ln \left(\frac{M_{a \mathrm{mir}}}{Q}\right)\right\}
$$

for $M_{a 0}$ and $M_{a \text { mir }}$ given by

$$
\begin{aligned}
M_{a 0} & =\frac{M_{0}}{1+\epsilon_{a}}, \\
M_{a \mathrm{mir}} & =M_{\mathrm{GUT}}\left(\frac{m_{3 / 2}}{M_{\mathrm{Pl}}}\right)^{\frac{\alpha}{2}\left(1+\epsilon_{a}\right)},
\end{aligned}
$$

where $\epsilon_{a} \equiv g_{0}^{2} \Delta_{a}$ parametrize the threshold corrections. The above shows that the unification of gaugino masses does not occur if the threshold corrections are nonuniversal. For universal $\epsilon_{a}$, the gauginos have a common mass at the mirage messenger scale, but its value is different from $M_{0}$ unless $\epsilon_{a}$ vanish. ${ }^{4}$ It is only when $\epsilon_{a}=0$ that mirage mediation effectively corresponds to pure moduli mediation transmitted at the mirage-messenger scale. Such correspondence is essential for reducing the electroweak fine-tuning because it allows to suppress the up-type Higgs soft mass at the mirage-messenger scale by taking an appropriate modular weight. We thus require that $\epsilon_{a}$ be at most around the loop factor, $1 / 8 \pi^{2}$, in size if any.

${ }^{4}$ Deflected mirage mediation [43-45] is an example of universal gauge-threshold corrections.
[1] H. P. Nilles, Supersymmetry, supergravity and particle physics, Phys. Rep. 110, 1 (1984).

[2] H. E. Haber and G. L. Kane, The search for supersymmetry: Probing physics beyond the standard model, Phys. Rep. 117, 75 (1985).

[3] P. W. Graham, D. E. Kaplan, and S. Rajendran, Cosmological Relaxation of the Electroweak Scale, Phys. Rev. Lett. 115, 221801 (2015).

[4] K. Choi, K. S. Jeong, and K.-i. Okumura, Phenomenology of mixed modulus-anomaly mediation in fluxed string compactifications and brane models, J. High Energy Phys. 09 (2005) 039.

[5] S. Kachru, R. Kallosh, A. D. Linde, and S. P. Trivedi, De Sitter vacua in string theory, Phys. Rev. D 68, 046005 (2003).

[6] K. Choi, A. Falkowski, H. P. Nilles, and M. Olechowski, Soft supersymmetry breaking in KKLT flux compactification, Nucl. Phys. B718, 113 (2005).

[7] K. Choi, K. S. Jeong, T. Kobayashi, and K.-i. Okumura, Little SUSY hierarchy in mixed modulus-anomaly mediation, Phys. Lett. B 633, 355 (2006).

[8] K. Choi, K. S. Jeong, T. Kobayashi, and K.-i. Okumura, $\mathrm{TeV}$ scale mirage mediation and natural little SUSY hierarchy, Phys. Rev. D 75, 095012 (2007).

[9] K. Choi and K. S. Jeong, Supersymmetry breaking and moduli stabilization with anomalous U(1) gauge symmetry, J. High Energy Phys. 08 (2006) 007.
[10] J. Kawamura and Y. Omura, Analysis of the TeV-scale mirage mediation with heavy superparticles, J. High Energy Phys. 11 (2017) 189.

[11] S. Krippendorf, H. P. Nilles, M. Ratz, and M. W. Winkler, Hidden SUSY from precision gauge unification, Phys. Rev. D 88, 035022 (2013).

[12] K. S. Jeong, Light higgsino for gauge coupling unification, Phys. Lett. B 769, 42 (2017).

[13] S. A. R. Ellis and J. D. Wells, High-scale supersymmetry, the Higgs boson mass, and gauge unification, Phys. Rev. D 96, 055024 (2017).

[14] S. D. Thomas and J. D. Wells, Phenomenology of Massive Vectorlike Doublet Leptons, Phys. Rev. Lett. 81, 34 (1998).

[15] H. Baer, V. Barger, and P. Huang, Hidden SUSY at the LHC: The light higgsino-world scenario and the role of a lepton collider, J. High Energy Phys. 11 (2011) 031.

[16] M. Berggren, F. Brümmer, J. List, G. Moortgat-Pick, T. Robens, K. Rolbiecki, and H. Sert, Tackling light higgsinos at the ILC, Eur. Phys. J. C 73, 2660 (2013).

[17] S. Nakamura, K.-i. Okumura, and M. Yamaguchi, Axionic mirage mediation, Phys. Rev. D 77, 115027 (2008).

[18] K. Choi, K. S. Jeong, W.-I. Park, and C. S. Shin, Thermal inflation and baryogenesis in heavy gravitino scenario, J. Cosmol. Astropart. Phys. 11 (2009) 018.

[19] H. Baer, V. Barger, P. Huang, D. Mickelson, A. Mustafayev, and X. Tata, Radiative natural supersymmetry: Reconciling 
electroweak fine-tuning and the Higgs boson mass, Phys. Rev. D 87, 115028 (2013).

[20] M. Carena, M. Quiros, and C. Wagner, Effective potential methods and the Higgs mass spectrum in the MSSM, Nucl. Phys. B461, 407 (1996).

[21] H. E. Haber, R. Hempfling, and A. H. Hoang, Approximating the radiatively corrected Higgs mass in the minimal supersymmetric model, Z. Phys. C 75, 539 (1997).

[22] M. Carena, H. Haber, S. Heinemeyer, W. Hollik, C. Wagner, and G. Weiglein, Reconciling the two loop diagrammatic and effective field theory computations of the mass of the lightest $C P$-even Higgs boson in the MSSM, Nucl. Phys. B580, 29 (2000).

[23] M. Carena, M. Olechowski, S. Pokorski, and C. Wagner, Electroweak symmetry breaking and bottom-top Yukawa unification, Nucl. Phys. B426, 269 (1994).

[24] M. Carena, S. Gori, N. R. Shah, and C. E. Wagner, A $125 \mathrm{GeV}$ SM-like Higgs in the MSSM and the $\gamma \gamma$ rate, J. High Energy Phys. 03 (2012) 014.

[25] M. Carena, J. Espinosa, M. Quiros, and C. Wagner, Analytical expressions for radiatively corrected Higgs masses and couplings in the MSSM, Phys. Lett. B 355, 209 (1995).

[26] ATLAS, CMS Collaborations, Combined Measurement of the Higgs Boson Mass in $p p$ Collisions at $\sqrt{s}=7$ and $8 \mathrm{TeV}$ with the ATLAS and CMS Experiments, Phys. Rev. Lett. 114, 191803 (2015).

[27] P. Athron, J.-h. Park, T. Steudtner, D. Stöckinger, and A. Voigt, Precise Higgs mass calculations in (non-)minimal supersymmetry at both high and low scales, J. High Energy Phys. 01 (2017) 079.

[28] B. Allanach and A. Voigt, Uncertainties in the lightest $C P$ even Higgs boson mass prediction in the minimal supersymmetric standard model: Fixed order versus effective field theory prediction, Eur. Phys. J. C 78, 573 (2018).

[29] H. Bahl, S. Heinemeyer, W. Hollik, and G. Weiglein, Theoretical uncertainties in the MSSM Higgs boson mass calculation, Eur. Phys. J. C 80, 497 (2020).

[30] ATLAS Collaboration, Search for squarks and gluinos in final states with jets and missing transverse momentum using $36 \mathrm{fb}^{-1}$ of $\sqrt{s}=13 \mathrm{TeV}$ pp collision data with the ATLAS detector, Phys. Rev. D 97, 112001 (2018).

[31] CMS Collaboration, Searches for physics beyond the standard model with the $M_{\mathrm{T} 2}$ variable in hadronic final states with and without disappearing tracks in proton-proton collisions at $\sqrt{s}=13 \mathrm{TeV}$, Eur. Phys. J. C 80, 3 (2020).
[32] ATLAS Collaboration, Search for top squarks in events with a Higgs or $Z$ boson using $139 \mathrm{fb}^{-1}$ of $p p$ collision data at $\sqrt{s}=13 \mathrm{TeV}$ with the ATLAS detector, Eur. Phys. J. C 80, 1080 (2020).

[33] CMS Collaboration, Search for top squark pair production using dilepton final states in pp collision data collected at $\sqrt{s}=13 \mathrm{TeV}$, Eur. Phys. J. C 81, 3 (2021).

[34] B.C. Allanach, sofTsusy: A program for calculating supersymmetric spectra, Comput. Phys. Commun. 143, 305 (2002).

[35] H. Baer, V. Barger, J. S. Gainer, P. Huang, M. Savoy, D. Sengupta, and X. Tata, Gluino reach and mass extraction at the LHC in radiatively-driven natural SUSY, Eur. Phys. J. C 77, 499 (2017).

[36] J. R. Ellis, K. Enqvist, D. V. Nanopoulos, and F. Zwirner, Observables in low-energy superstring models, Mod. Phys. Lett. A 01, 57 (1986).

[37] R. Barbieri and G. Giudice, Upper bounds on supersymmetric particle masses, Nucl. Phys. B306, 63 (1988).

[38] S. Dimopoulos and G. Giudice, Naturalness constraints in supersymmetric theories with nonuniversal soft terms, Phys. Lett. B 357, 573 (1995).

[39] H. Baer, V. Barger, and D. Mickelson, How conventional measures overestimate electroweak fine-tuning in supersymmetric theory, Phys. Rev. D 88, 095013 (2013).

[40] H. Baer, V. Barger, and D. Sengupta, Mirage mediation from the landscape, Phys. Rev. Research 2, 013346 (2020).

[41] H. Baer, V. Barger, S. Salam, D. Sengupta, and X. Tata, The LHC higgsino discovery plane for present and future SUSY searches, Phys. Lett. B 810, 135777 (2020).

[42] S. Raby, M. Ratz, and K. Schmidt-Hoberg, Precision gauge unification in the MSSM, Phys. Lett. B 687, 342 (2010).

[43] L. L. Everett, I.-W. Kim, P. Ouyang, and K. M. Zurek, Deflected Mirage Mediation: A Framework for Generalized Supersymmetry Breaking, Phys. Rev. Lett. 101, 101803 (2008).

[44] L. L. Everett, I.-W. Kim, P. Ouyang, and K. M. Zurek, Moduli stabilization and supersymmetry breaking in deflected mirage mediation, J. High Energy Phys. 08 (2008) 102.

[45] K. Choi, K. S. Jeong, S. Nakamura, K.-I. Okumura, and M. Yamaguchi, Sparticle masses in deflected mirage mediation, J. High Energy Phys. 04 (2009) 107. 